\title{
Increased D-arabinitol/creatinine ratio in sera of patients with Behçet's disease during an active phase
}

\author{
Seiji Hayasaka, Sachiko Noda, Tomoichi Setogawa
}

\begin{abstract}
The ratios of D-arabinitol (a major metabolite of Candida species) to creatinine were examined in 46 sera of 40 patients with ocular inflammatory disease and in 50 age-matched normal controls ( 25 men and 25 women). The mean ratio in patients with endogenous Candida endophthalmitis was higher than in the normal controls. Seven samples taken from patients with Behçet's disease during an active phase showed higher values than in the controls. Fourteen patients with Behçet's disease in remission showed D-arabinitol/ creatinine values within normal range. In four patients with sarcoidosis, in two patients with Vogt-Koyanagi-Harada disease, and in 16 patients with bilateral uveitis of an unknown cause, the ratios were within normal range. It is possible that $D$-arabinitol, or a metabolite of Candida species, may be related in part to the active phase of Behçet's disease.

(Brf Ophthalmol 1993; 77: 39-40)
\end{abstract}

The aetiology of Behçet's disease remains unresolved. Namba $e t a l^{1}$ and Mizushima ${ }^{2}$ have recently described that some components of Streptococcus may be a possible exogenous factor in the pathogenesis of the disease. Cooper $e t a l^{3}$ have reported that the disease may be triggered by childhood infection. Increased levels of D-arabinitol, a major metabolite of Candida species, have been reported in sera of patients with invasive candidiasis and endogenous Candida species endophthalmitis. ${ }^{47}$ We examined D-arabinitol/creatinine ratios in the sera of patients with several ocular inflammatory diseases to investigate a possible factor in the pathogenesis of Behçet's disease.

\section{Subjects and methods}

\section{SUBJECTS}

Department of Ophthalmology, Shimane Medical University, Izumo, Japan S Hayasaka S Noda

T Setogawa

Correspondence to: Dr Seiji Hayasaka, Department of Ophthalmology, Shimane Medical University, Izumo Shimane 693, Japan.

Accepted for publication 1 October 1992
We examined 40 patients ( 19 men and 21 women) with ocular inflammatory disease who were seen at the eye clinic of Shimane Medical University Hospital. They ranged in age from 32 to 67 years (mean 51 years). All patients had cells in the anterior chamber and/or vitreous at the time or, several months before, we obtained samples of their blood.

Two patients with endogenous Candida endophthalmitis (one of them has been described previously ${ }^{+}$) had intracameral and vitreous cells and Candida albicans cultured from a catheter tip. Patients with Behçet's disease who had an ocular lesion, an oral ulceration, or other disorders were classified as the complete or incomplete type, according to the Japanese criteria, ${ }^{8}$ or were fitted to the International Study Group criteria. ${ }^{9}$ Their ages ranged from 34 to 61 years (mean, 48 years). These patients were divided according to active and remission phases of the disease, based on the presence or absence of intracameral cells at the time of obtaining the blood specimens. Patients with Behçet's disease in remission exhibited intracameral cells several months beforehand.

Sarcoidosis was diagnosed by iris nodules, biopsy findings of the conjunctiva, increased level of serum angiotensin converting enzyme, and bilateral hilar lymphadenopathy on $x$-ray film. Diagnosis of Vogt-Koyanagi-Harada disease was based on the bilateral onset of exudative uveitis, tinnitus, vitiligo, and meningitis. Patients with uveitis of an unknown cause had intracameral and vitreous cells in both eyes, but had no evidence of a specific type of the disease or peculiar laboratory findings, including serum titres for Toxoplasma gondii, or microhaemagglutination for Treponema pallidum. Of 16 subjects with uveitis of an unknown cause, six had anterior uveitis, four had posterior uveitis, and the others had panuveitis. Their ages ranged from 32 to 67 years (mean 51 years).

The patients were classified into two groups: with and without systemic corticosteroid treatment.

Individuals ( 25 men and 25 women) without apparent systemic or ocular inflammatory disease served as the normal controls. They ranged in age from 32 to 67 years (mean, 50 years).

Excluded from the present study were patients with diabetes mellitus, those with leukaemia, and those with Behçet's disease treated with cyclosporin.

\section{Methods}

A $2.0 \mathrm{ml}$ aliquot of peripheral blood was obtained from a cubital vein, and the serum was separated by centrifugation. D-Arabinitol values were examined by the enzymatic fluorometric method of Soyama and Ono. ${ }^{10}$ Creatinine levels were analysed by the method of Heinegard and Tiderstrom." The assays were run in duplicate, and the mean value was employed in each blood sample.

The data were analysed using Student's $t$ test. 
Table 1 D-Arabinitol/creatinine ratio in sera

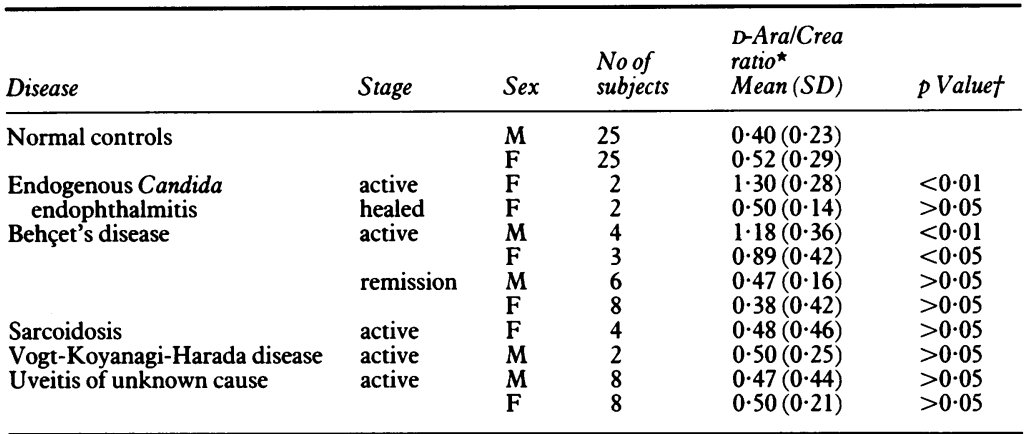

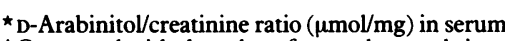

†Compared with the value of normal controls in each sex.

A p value less than 0.05 was considered statistically significant.

\section{Results}

D-Arabinitol/creatinine ratios $(\mu \mathrm{mol} / \mathrm{mg})$ in sera of 50 normal individuals and in 46 samples of patients with ocular inflammatory disease were prepared (Table 1). The samples of sera from 25 normal men (ages 32 to 67 years) showed ratios from 0 to 0.8 (mean (SD) $0.40(0.23)$ ). The samples of será from 25 normal women (ages 34 to 66 years) revealed ratios from 0 to $1 \cdot 1$ (mean (SD) $0.52(0.29)$ ).

The mean ratio in two patients with endogenous Candida endophthalmitis was $1 \cdot 30$ in the active stage and was 0.50 in remission. Samples from four men and three women with Behçet's disease in the active phase showed higher values than in the normal controls. The 14 samples from patients with Behçet's disease in remission showed ratios within normal range. Four patients with sarcoidosis, two patients with Vogt-Koyanagi-Harada disease, and 16 patients with uveitis of an unknown cause showed ratios within normal range.

Eight patients who were being given systemic corticosteroid treatment showed values that were within normal range.

\section{Discussion}

We reported previously the increased value of $D$ arabinitol in sêrum of a patient with endogenous Candida endophthalmitis. ' In the present study, the $D$-arabinitol/creatinine ratio was measured in 40 patients with ocular inflammatory disease and in 50 age-matched normal controls. Because the clearance of D-arabinitol from the kidney reportedly has been the same as that of creatinine, the ratio may be expressed independent of the condition of the kidney..$^{5}$ The D-arabinitol/ creatinine ratio in normal men had differed from that in normal women. ${ }^{5}$ We therefore compared the ratio in each sex in the present study. The ratios in normal controls in our study were similar to those reported by Soyama and Ono. ${ }^{5}$

High ratios have been reported in patients with diabetes mellitus and in those with leukaemia. ${ }^{5}$ Therefore, such patients were excluded from the present study.

The ratios in patients with Behçet's disease in the active phase were characteristically increased in our present study. Though an association of HLA BW-51 and Behçet's disease has been proposed,,$^{12}$ the exact pathogenesis of the disease is uncertain. Namba et al ${ }^{\prime}$ and Mizushima ${ }^{2}$ have recently indicated that some components of Streptococcus may be a possible exogenous factor in the pathogenesis of the disease. Copper et al have reported that the disease may be triggered by childhood infection. It is possible that Candide species may be common in the gastrointestinal tract of apparently healthy subjects. Candida infection of anti-Candida immune status may be related in part to the active phase of Behçet's disease.

This work was supported in part by a grant from the Behçet' Disease Research Committee of the Ministry of Health and Welfare of Japan.

1 Namba K, Ueno T, Okita $M$. Behçet's disease and streptococcal infection. f pn f Ophthalmol 1986; 30: 385-401.

2 Mizushima Y. Problems and significance of Streptococcus in the pathogenesis of Behçet's disease. Saishin-igaku 1988; 43: 289-93.

3 Cooper C, Pippard EC, Sharp H, Wickham C, Chamberlain MA, Barker DIP. Is Behcet's disease triggered by childhood infection. Ann Rheum Dis 1989; 48: 421-3.

4 Kiehn TE, Bernard EM, Gold JWM, Armstrong D. Candidiasis. Detection by gas-liquid chromatography of $\mathrm{D}-$ arabinitol, a fungal metabolite in human serum. Science 1979; 206: 577-80

5 Soyama K, Ono E. D-Arabinitol concentrations and Darabinitol/creatinine ratios in serum obtained from patien with invasive candidiasis. Rinsho Byori 1987; 35: 1039-42.

6 Wong B, Brauer KL. Enantioselective measurement of funga D-arabinitol in the sera of normal adults and patients with candidiasis. $\mathcal{f}$ Clin Microbiol 1988; 26: 1670 .

7 Hayasaka S, Noda S, Setogawa T. Endogenous Candida species endophthalmitis associated with increased levels of D-arabinitol in serum and vitreous. Am F Ophthalmol 1991; 111: $379-80$.

8 Behçet's Disease Research Committee of Japan. Behçet's disease: guide to diagnosis of Behçet's disease. Ipn $f$ Ophthalmol 1974; 18: 291-4.

9 International Study Group for Behcet's Disease Criteria for diagnosis of Behçet's disease. Lancet 1990; 335: 1078-80.

10 Soyama K, Ono E. Enzymatic fluorometric method for th determination of D-arabinitol in serum by initial rate analysis. Clin Chim Acta 1985; 149: 149-54

11 Heinegard D, Tiderstrom G. Determination of serum creatinine by a direct colorimetric method. Clin Chim Act 1973; 43: 305-10.

12 Ohno S, Asanuma T, Sugiura S. HLA-BW 51 and Behçet's disease. $\mathcal{F A M A}$ 1978; 240: 529 . 